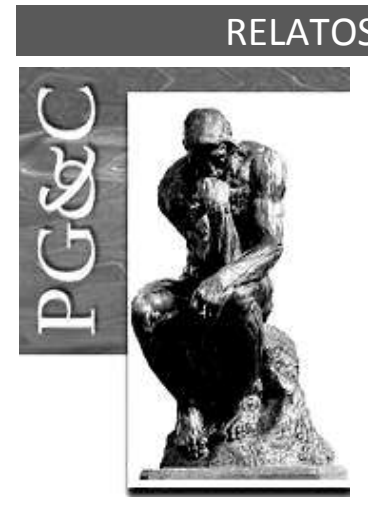

\title{
SMART CAMPUS E A GESTÃO DA INFORMAÇÃO: APLICABILIDADES NA UNIVERSIDADE FEDERAL DE CAMPINA GRANDE
}

\author{
Lucilene Klenia Rodrigues Bandeira \\ Doutora em Administração pela Université Grenoble Alpes, França. \\ Professora da Universidade Federal da Paraíba, Brasil. \\ E-mail: klenia.bandeira@gmail.com \\ Adelaide Helena Targino Casimiro \\ Doutoranda em Ciência da Informação pela Universidade Federal da \\ Paraíba, Brasil. Professora da Universidade Federal do Rio Grande do \\ Norte, Brasil. \\ E-mail: adelaide helena@hotmail.com
}

Ediene Souza de Lima

Mestra em Ciência da Informação pela Universidade Federal da Paraíba, Brasil. Analista Técnica do Sebrae Paraíba, Brasil.

E-mail: dienelim@hotmail.com

\begin{abstract}
Resumo
Objetiva analisar as práticas de Smart Campus da Universidade Federal de Campina Grande (UFCG) em relação às etapas de Gestão da Informação (GI) do modelo de Choo. Smart Campus é uma área de pesquisa em que se usam tecnologia e infraestrutura para apoiar e melhorar seus processos nos serviços em instituições de ensino superior, auxiliando o ensino, a extensão e a pesquisa. A Gestão da Informação traz benefícios na administração da informação de forma integrada, coerente, eficiente e eficaz; facilita a tomada de decisão e eleva ao máximo a qualidade, a disponibilização, a utilização e o valor da informação. A UFCG usa as técnicas de Smart Campus desde 2019, com o objetivo de integrar a Internet das coisas à gestão do campus, ao ensino e às colaborações intra e interorganizacionais. Este estudo, de natureza exploratória, descritiva, com abordagem qualitativa e natureza documental direta, e para cujo desenvolvimento utilizamos a técnica de Análise de Conteúdo e aplicamos um questionário ao gestor do projeto de implantação do Smart Campus na UFCG, indicou que as aplicabilidades do Smart Campus dialogam com o modelo de GI de Choo. A UFCG se preocupa em identificar as necessidades de informação, que é adquirida por meio da interação entre professores e alunos em redes sociais e distribuída por meio de reuniões semanais com grupos de interesses, página da Internet e redes sociais. Concluímos que essa instituição não pratica as etapas de uso de informação e avaliações de desempenho, embora existam iniciativas em andamento.
\end{abstract}

Palavras-chave: Smart Campus. Gestão da Informação. Tecnologia da Informação. Universidade Federal de Campina Grande.

SMART CAMPUS AND INFORMATION MANAGEMENT: APPLICATIONS AT UNIVERSIDADE FEDERAL DE CAMPINA GRANDE

\begin{abstract}
Aims to analyze the Smart Campus practices of the Federal University of Campina Grande (UFCG) in relation to the Information Management (IM) stages of the Choo model. Smart Campus is a research area in which technology and infrastructure are used to support and improve their service processes in higher education institutions, assisting teaching, extension and research. Information Management brings benefits in the administration of information in an integrated, coherent, efficient and effective
\end{abstract}

Perspectivas em Gestão \& Conhecimento, João Pessoa, v. 10, número especial, p.23-37, mar. 2020. DOI: http://dx.doi.org/10.21714/2236-417X2020v10nep23

http://periodicos.ufpb.br/ojs2/index.php/pgc. ISSN: 2236-417X. Publicação sob Licença (cc) EY-NC-ND 
way; it facilitates decision making and maximizes the quality, availability, use and value of information. UFCG has been using Smart Campus techniques since 2019, with the objective of integrating the Internet of things to campus management, teaching and the intra- and inter-organizational collaborations. This study has an exploratory and descriptive nature, with a qualitative approach and a direct documental nature, and for whose development we used the Content Analysis technique and applied a questionnaire to the Smart Campus implementation project manager at UFCG, indicated that the Smart Campus applications combines with the Choo's IM model. UFCG is concerned with identifying information needs, which is acquired through interaction between teachers and students on social networks and distributed through weekly meetings with interest groups, the websites and social networks. We concluded that this institution does not practice the Choo's IM model steps of information's use and performance evaluations, although there are initiatives in progress.

Keywords: Smart Campus. Information Management. Information Technology. Federal University of Campina Grande.

\section{CONSIDERAÇÕES INICIAIS}

Com o contínuo desenvolvimento das Tecnologias da Informação e Comunicação (TICs), as pessoas estão passando, gradativamente, da era digital para a era inteligente. Como uma das tecnologias emergentes mais representativas dessa era, a inteligência artificial está mudando silenciosamente nossas vidas a um ritmo sem precedentes (LIANG; CHEN, 2018). Nosso estilo de vida vem sendo impactado e impactando o desenvolvimento dessas tecnologias. O uso contínuo de tecnologias móveis que aproximam as pessoas e reduzem o tempo de resposta entre elas é um exemplo disso. Nesse cenário, a informação é um elemento primordial, que estimula os fenômenos sociais e, consequentemente, os processos decisórios das instituições públicas ou privadas, e a base para o planejamento e a execução das ações com foco na inovação de produtos e serviços, tendo como suporte a Tecnologia da Informação.

Nas universidades públicas, os desafios atuais relacionados à Ciência e à Tecnologia refletem-se nas atividades dos Institutos de Educação Superior (IES), onde a aplicação das práticas tecnológicas e a realidade da tendência digital são crescentes. Logo, buscamos compreender conceitos ligados ao Smart Campus e sua aplicabilidade, especificamente na Universidade Federal de Campina Grande (UFCG). Kwok (2015) define o Smart Campus como a integração dinâmica do mundo digital e do mundo físico, em que os dados de várias fontes são coletados automaticamente em tempo real. Sob a ótica de Choo (2003), a análise da informação que perpassa esse processo é feita por meio das seguintes etapas do modelo de Gestão da Informação (GI): 1) identificação das necessidades; 2) aquisição; 3) organização e armazenamento; 4) desenvolvimento de produtos e serviços; 5) distribuição; e 6) uso. Portanto, esta pesquisa foi fundamentada nos conceitos de Smart Campus e suas práticas, tendo como base as etapas da Gestão da Informação, com o objetivo de saber quais os mecanismos que esse processo propõe para uma gestão efetiva dos recursos informacionais.

A questão que norteou esta pesquisa foi a seguinte: Considerando o modelo Smart Campus adotado pela UFCG, quais as práticas alinhadas ao modelo de Gl de Choo (2003)? Para responder a essa pergunta, objetivamos analisar as práticas de Smart Campus da UFCG, em relação às etapas de Gestão de Informação do modelo de Choo (2003), considerado o melhor para esta pesquisa, porque suas etapas são bem definidas e têm um alto grau de aplicabilidade organizacional, em especial, as que utilizam o Smart Campus como insumo estratégico. As etapas de GI são comportamentos apropriados a partir dos quais surgem as demandas de

Perspectivas em Gestão \& Conhecimento, João Pessoa, v. 10, número especial, p. 23-37, mar. 2020. 
informação e a viabilização do processo de Gestão da Informação. Destaca-se, ainda, a necessidade de administrar o fluxo de informações em rede.

Nesse contexto, ressaltamos o papel fundamental do modelo Smart Campus relacionado às etapas da Gestão da Informação para contribuir com o avanço da Ciência e da Educação, cujo foco é no capital intelectual e apresenta-se como um fator indispensável para a inovação. O capital intelectual constitui a matéria intelectual, como o conhecimento, a informação, a propriedade intelectual e as experiências que podem ser utilizadas para gerar riqueza (STEWART, 1998).

As mudanças decorrentes do desenvolvimento tecnológico contribuem para melhorar a qualidade da informação nas IES, quando pode ser apresentada em tempo hábil para a tomada de decisão. Nesse sentido, o objetivo deste artigo é de avaliar as ações de Gestão da Informação no Smart Campus da UFCG. Entende-se que um modelo de gestão que adota a tecnologia da informação como base para atingir os objetivos organizacionais deve pensar na gestão da informação como um elemento estratégico para que sua aplicabilidade seja eficaz.

\section{SMART CAMPUS: um conceito emergente}

Com o rápido desenvolvimento da computação em nuvem, big data e internet das coisas, a tecnologia da informação avançada vem sendo, gradualmente, integrada ao setor educacional, fazendo com que o nível de informatização da universidade também melhore constantemente (YANG et al., 2018). Nesse contexto, surgem os Smart Campus, cujo conceito surgiu do conceito de smart cities, que é a integração dinâmica do mundo digital e do mundo físico, em que os dados de várias fontes são coletados automaticamente em tempo real (KWOK, 2015). O campus passa a ser comparado com uma cidade que apresenta os mesmos desafios de gestão uma cidade convencional.

Muitos autores apresentam propostas para o Smart Campus, que são específicas para determinado problema ou mais globais e envolvem também o ensino. Ying et al. (2012) propõem a informatização do campus, por meio de um modelo inteligente, e discutem sobre as principais tecnologias baseadas na ideia de técnica orientada para objetos e encapsulamento de serviços. Esse modelo pode suportar a maioria das atividades do campus, como controle de acesso, comércio eletrônico e serviços eletrônicos. Ai-Min et al. (2018) analisam as dificuldades de integrar vários tipos de serviços de dados e projetam um algoritmo de fusão de dados.

Shaojie et al. (2018) projetaram um algoritmo de previsão de desempenho dos estudantes. O método proposto é comparado com quatro algoritmos. Os resultados demonstraram que o método desenvolvido melhora o desempenho. Xin et al. (2018) utilizaram uma combinação de algoritmos para avaliar o desempenho dos professores e estabeleceram um esquema de coleta de dados de educação no Smart Campus e a plataforma de armazenamento, a qual fornece um modelo de referência para a construção de Smart Campus. Eles analisam as principais características do Smart Campus a partir das perspectivas de tecnologia, negócios e modo de construir e propõem um modelo de arquitetura de Smart Campus.

Smart Campus é uma área de pesquisa interessante, nova e emergente, em que se usam tecnologia e infraestrutura para apoiar e melhorar seus processos nos serviços do campus, no ensino, na aprendizagem e na pesquisa (ZHIHONG et al. 2018). A gestão do campus dispõe de uma plataforma inteligente que promove uma gestão, em tempo real, dos principais problemas enfrentados pelos gestores no cotidiano do campus e a captação de dados importantes que servirão como base para a memória de cálculo dos recursos e para promover as aplicações de ações estratégicas. Segundo Kar e Gupta (2015), um Smart Campus

Perspectivas em Gestão \& Conhecimento, João Pessoa, v. 10, número especial, p. 23-37, mar. 2020. 
fundamenta-se em três pilares: infraestrutura, operações e pessoas. De acordo com esses autores, as principais características desse modelo são:

- Automação habilitada para a tecnologia inteligente;

- Serviços integrados via dashboards;

- Eficiência energética e hídrica (sistemas inteligentes de distribuição, medidores inteligentes etc.);

- Criatividade e inovação por meio da colaboração;

- Resultados nas melhores práticas.

No Brasil, podemos citar alguns modelos de Smart Campus em instituições privadas e públicas. Segundo Romano, Pinto e Pacheco (2016), em 2014, o Centro Universitário FACENS submeteu um projeto ao Global Entrepreneurship Lab do Massachusetts Institute of Technology (MIT), Sloan-School of Management e foi contemplada com uma assessoria para criar o seu Smart Campus. No âmbito dos Institutos de Ensino Superior (IES), em especial, das universidades federais, podemos citar iniciativas mais recentes, como a da Universidade Federal do Rio Grande do Norte (UFRN), a da Universidade Federal do Espírito Santo (UFES), a da Universidade Federal de Santa Catarina (UFSC) e a da Universidade Estadual de Campinas (Unicamp), que criaram seus Smart Campus desde 2016, e da Universidade Federal de Campina Grande, que criou o seu em 2019.

Os princípios do Smart Campus são a colaboração intra e interinstitucional, o desenvolvimento sustentável, a eficiência, a proteção ambiental, a transparência e a justiça social, e sua plataforma envolve o uso e o desenvolvimento de conceitos e tecnologias. Os eixos estratégicos do Smart Campus são material de consumo, energia elétrica, água, esgoto, resíduos sólidos, qualidade de vida, compras, contratações sustentáveis, mobilidade, segurança, urbanização, paisagismo, acessibilidade, educação ambiental, uso e ocupação de espaço e outras ações.

Todos os exemplos citados contemplam os pilares citados por Kar e Gupta (2015). Entretanto, não tivemos informações suficientes para afirmar se existem dimensões privilegiadas ou negligenciadas. A emergência dos modelos ainda não oferece dados suficientes para serem comparados entre as instituições. No Quadro 1 apresentamos alguns conceitos de Smart Campus encontrados na literatura:

Quadro 1 - Conceitos de Smart Campus

\begin{tabular}{|c|c|}
\hline Autores & Conceitos \\
\hline Ying et al. (2012) & $\begin{array}{l}\text { O campus digital é baseado em rede e utiliza meios e ferramentas de informação } \\
\text { avançados. Smart Campus é um estágio avançado na construção da } \\
\text { informatização do campus, onde a vida cotidiana pode ser mais conveniente. }\end{array}$ \\
\hline Yang et al. (2018) & $\begin{array}{l}\text { Smart Campus é um campus intelectualizado, em que se aprende e se vive em um } \\
\text { ambiente integrado e baseado na internet das coisas. É uma plataforma integrada } \\
\text { de serviços de informação aberta, inovadora, colaborativa e inteligente. }\end{array}$ \\
\hline Xin et al. (2018) & $\begin{array}{l}\text { As características do campus inteligente incluem conscientização ambiental } \\
\text { abrangente, conexão de rede contínua, suporte maciço a dados, ambiente de } \\
\text { aprendizado aberto e serviços personalizados para professores e alunos. }\end{array}$ \\
\hline Dong et al. (2016) & $\begin{array}{l}\text { Um Smart Campus deve apresentar três características: a percepção precisa do } \\
\text { contexto e o acesso onipresente à rede; a alocação eficiente de recursos; e a } \\
\text { tomada de decisão inteligente baseada em princípios objetivos. }\end{array}$ \\
\hline Li (2018) & $\begin{array}{l}\text { A essência de um campus inteligente é uma plataforma virtual para troca de } \\
\text { informações. A tecnologia da Internet das coisas é necessária para ser usada ao } \\
\text { máximo e construir um sistema de rede gigante que pode fornecer pesquisa de } \\
\text { informações, troca de recursos e outros serviços para professores e alunos e, } \\
\text { finalmente, fazer o trabalho de gestão do campus inteligente e em tempo real. }\end{array}$ \\
\hline
\end{tabular}

Perspectivas em Gestão \& Conhecimento, João Pessoa, v. 10, número especial, p. 23-37, mar. 2020. 


\begin{tabular}{|l|l|}
\hline \multirow{5}{*}{ Feng et al. (2018) } & $\begin{array}{l}\text { O campus inteligente é uma nova etapa da construção de tecnologias de } \\
\text { informação em universidades. Suas características incluem onipresença de alta } \\
\text { velocidade da Internet, Internet das Coisas, interconexão contínua, consciência } \\
\text { ambiental, ampla popularização e aplicação de terminais inteligentes e } \\
\text { aprendizagem colaborativa aberta no ambiente de pesquisa e no de trabalho. O } \\
\text { campus inteligente está intimamente integrado às novas tecnologias da Internet, } \\
\text { que usam computação em nuvem, virtualização e a Internet das Coisas para } \\
\text { mudar as formas de interagir, de transferir conhecimentos e de compartilhar } \\
\text { recursos entre os usuários do campus. }\end{array}$ \\
\hline
\end{tabular}

Fonte: Elaboração própria (2019)

Os estudos sobre Smart Campus se concentram nas áreas de tecnologia, com foco no desenvolvimento de algoritmos complexos para atender a uma necessidade específica do campus, como cibersegurança big data, redes mais velozes etc. Percebemos que as definições de Smart Campus se complementam e focam na tecnologia da internet das coisas em um ambiente conectado que promove a inovação e a colaboração em vários níveis e categorias dentro do campus, como: ensino, aprendizagem, sustentabilidade, gestão, segurança dos dados etc. As TICs formam uma dimensão importante do Smart Campus, porém a gestão da informação é um fator estratégico nesse modelo, mas não foi mencionada nos conceitos dos trabalhos identificados. Entendemos que a informatização do campus é importante e necessária, mas não se passa do digital para o inteligente apenas com o uso da tecnologia, portanto, a gestão da informação é essencial nesse processo assim como as pessoas que participarão desse processo.

\section{GESTÃO DA INFORMAÇÃO: MODELO DE CHUN WEI CHOO}

Para Le Coadic (2004, p.3), "a informação é uma medida da organização de um sistema que [...] comporta um elemento de sentido". Já Drucker (1988) a define como um conjunto de dados que têm sentido e relevância para determinado contexto ou pessoa. Portanto, podemos afirmar que a informação é relevante para criar e desenvolver as relações individuais, institucionais e/ou sociais e essencial para caracterizar o humano como um sujeito dotado de raciocínio. Ela identifica as particularidades dos sujeitos, transmite mensagens entre os indivíduos e a sociedade e faz parte dos procedimentos organizacionais.

Já Casimiro (2019, p.41) assevera que a informação determina as fundações para o processo de conhecimento. Logo, esse é um recurso inerente ao indivíduo e carece da informação para evoluir. É a forma como o sujeito cognoscente "interpreta as informações captadas do ambiente, atribui-Ihes um sentido e está intimamente ligada ao contexto do tempo, do espaço e da vivência particulares de cada um. É uma relação entre o sujeito e o objeto." Afinal, o conjunto de informações internalizadas e contextualizadas pelo gestor, por meio do conhecimento, terá alto impacto na tomada de decisão e na gestão de sua instituição.

De acordo com Araújo (2014), muitos pesquisadores da área de Ciência da Informação (Cl) entendem a atualidade como parte integrante da 'Sociedade da Informação e do Conhecimento'. Os estudos sobre Gestão da Informação e do Conhecimento (GIC) contribuem para que os gestores compreendam cientificamente o planejamento, a organização, a direção e o controle adequado das organizações, de modo a utilizar os conhecimentos explícitos (ou informação) e os tácitos (ou conhecimento) de cada um deles como recursos organizacionais importantes para o bom funcionamento da organização e seu crescimento (CASIMIRO, 2019).

Em Leite (2011), a gestão é reconhecida como o ato de planejar, organizar, dirigir e controlar. Em alguns casos, é considerada como sinônimo de administração. Os estudos que têm como base os princípios da Gestão da Informação (GI) têm ênfase na administração dos

Perspectivas em Gestão \& Conhecimento, João Pessoa, v. 10, número especial, p. 23-37, mar. 2020. 
recursos físicos de uma organização. Nesse contexto, a Gl é uma forma que vem sendo utilizada pelas organizações para administrar a informação para que ela seja aproveitada da melhor forma possível, a fim de auxiliar o processo de tomada de decisão.

A GI é composta de etapas que, se forem utilizadas corretamente, podem trazer benefícios como a gerência da informação, de forma integrada, coerente, eficiente e eficaz; a utilização em seu máximo potencial, para que a informação chegue às pessoas certas, no local correto, no tempo certo, no formato adequado e com o custo certo, o que poderá facilitar a tomada de decisão e elevar ao máximo a qualidade, a disponibilização, a utilização e o valor da informação (LEITE, 2011).

Vários autores criaram modelos para otimizar o entendimento das etapas ligadas ao processo de Gl. Na literatura científica sobre $\mathrm{Cl}$, os mais conhecidos são os modelos de McGee e de Prusak (1994), o de Davenport (2002) e o de Choo (2003), cujas etapas estão elencadas no Quadro 2:

Quadro 2 - Modelos de Gestão da Informação

\begin{tabular}{|c|c|c|}
\hline Choo (2003) & Davenport (2002) & McGee e Prusak (1994) \\
\hline $\begin{array}{l}\text { Identificação das necessidades } \\
\text { de informação }\end{array}$ & $\begin{array}{l}\text { Determinação de exigências } \\
\text { de informação }\end{array}$ & $\begin{array}{l}\text { Identificação de necessidades e } \\
\text { requisitos de informação }\end{array}$ \\
\hline Aquisição de informação & Obtenção de informação & Aquisição e coleta de informação \\
\hline $\begin{array}{l}\text { Organização e armazenamento } \\
\text { da informação }\end{array}$ & Distribuição da informação & $\begin{array}{l}\text { Classificação, armazenamento, } \\
\text { tratamento e apresentação da } \\
\text { informação }\end{array}$ \\
\hline $\begin{array}{l}\text { Desenvolvimento de produtos } \\
\text { informacionais e serviços }\end{array}$ & \multirow{3}{*}{ Utilização da informação } & $\begin{array}{l}\text { Desenvolvimento de produtos e } \\
\text { serviços de informação }\end{array}$ \\
\hline Distribuição da informação & & $\begin{array}{l}\text { Distribuição e disseminação da } \\
\text { informação }\end{array}$ \\
\hline Uso da informação & & Análise e uso da informação \\
\hline
\end{tabular}

Fonte: Adaptado de Choo (2003), Davenport (2002) e McGee e Prusak (1994)

Como indicado no Quadro 2, os autores tiveram a liberdade de criar seus modelos de acordo com suas experiências e com seus conhecimentos. No entanto, para atender às exigências deste trabalho, consideramos que o modelo de Choo (2003) é o que mais bem se enquadra, porque tem etapas bem definidas e uma rica literatura de aplicabilidade nas mais variadas organizações, e porque suas etapas são as que mais atendem às exigências de aplicabilidade de Smart Campus em instituições de ensino público, em especial, a Universidade Federal de Campina Grande, objeto desta pesquisa.

O modelo é dividido em seis etapas, que, por sugestão do autor, devem ser aplicadas de modo contínuo e cíclico, para que, depois de concluída a etapa 'uso da informação', seja iniciada a de 'identificação das necessidades de informação', e assim sucessivamente. Choo (2003) espera que, durante cada etapa, sejam feitos os seguintes procedimentos:

1. Identificar as necessidades de informação: apontar o problema, os resultados esperados e onde é possível procurar as soluções. As necessidades de informação são incertas, dinâmicas e multifacetadas, e uma especificação completa só é possível em uma rica representação de todo o ambiente em que a informação é usada;

2. Adquirir informações: a seleção e o uso das fontes para adquirir informações precisam ser planejados e continuamente monitorados e avaliados como qualquer outro recurso vital da organização. A variedade de informações deve ser administrada de modo que as informações coletadas reflitam a complexidade do ambiente, sem sobrecarregar os usuários com excesso de informação. 
3. Organizar e armazenar a informação: criar um sistema digital ou físico, com flexibilidade necessária para captar as informações, apoiar as múltiplas visões que os usuários têm dos dados, conectar itens que são funcionais ou logicamente relacionados e permitir que os usuários explorem padrões e conexões;

4. Desenvolver produtos e serviços de informação: os produtos de informação devem ter uma abordagem de agregação de valor, na qual os sistemas, os produtos e os serviços de informação são desenvolvidos como qualidades que agregam valor à informação que está sendo processada, com o objetivo de ajudar o gestor a tomar melhores decisões, a perceber melhor as situações e, em última instância, a escolher ações mais eficazes;

5. Distribuir a informação: as informações se disseminam na organização para atingir as pessoas certas, no momento, lugar e formato adequados. A distribuição da informação promove sua partilha e sua recuperação;

6. Usar a informação: as organizações utilizam a informação com três finalidades: a de criar significado, construir conhecimentos e tomar decisões.

A Gestão da Informação, de acordo com Choo (2003), relaciona-se ao conceito de Gestão do Conhecimento, especialmente quando trata da Teoria Organizacional que reflete sobre as "organizações aprendizes". Choo (2003, p.403) recomenda que [...] a Gestão da Informação seja vista como a administração de uma rede de processos que adquirem, criam, organizam, distribuem e usam a informação.

Logo, para o autor, a eficiência desse processo se deve à execução das etapas do modelo de GI com a identificação das necessidades de informação; aquisição da informação; organização e armazenamento da informação; desenvolvimento de produtos e serviços de informação; distribuição da informação e uso da informação.

Este artigo contribui com a compreensão de conceitos ligados ao Smart Campus e sua aplicabilidade, especificamente na Universidade Federal de Campina Grande. A análise da informação que perpassa esse processo se dá a partir das etapas do modelo de $\mathrm{Gl}$, expressas anteriormente, sob a ótica de Choo (2003). Nas instituições de ensino superior, a realidade atual conduz à agilidade dos processos, à necessidade de integração, à conectividade e à simultaneidade, o que gera um impacto nos resultados e na tomada de decisão.

Nesse cenário, as ferramentas de tecnologia da informação são necessárias para analisar dados e transformá-los em informações estratégicas. A obtenção de informações primordiais norteia a resolução de problemas e a eficiência na tomada de decisões diante dos desafios institucionais. Considerando a discussão sobre Smart Campus e sua relação com as etapas de Gestão da Informação, sob a ótica de Choo (2003), e com os objetivos do estudo em questão, evidenciamos argumentos sobre a eficiência dos recursos informacionais e a inovação com foco no ensino, na aprendizagem e na extensão, por meio do acesso, do uso e do compartilhamento da informação e sua relação com a estratégia e a gestão da informação, a qual é essencial em ambientes institucionais.

Para Davenport (2002), a adoção de uma abordagem "ecológica" pode contribuir para melhorar o processo de $\mathrm{Gl}$, ao se observar o processo a partir do ambiente que envolve a arquitetura e a tecnologia da informação. Isso significa que se deve encarar o processo do ponto de vista do ambiente como um todo: arquitetura, estratégias, políticas e comportamentos ligados à informação; processos de trabalho e pessoas. A abordagem ecológica compreende a integração de diversos tipos de informação (estruturada, não estruturada, automatizada, não automatizada, textos, áudio, vídeo etc.); o reconhecimento das tendências à mudança; ênfase na observação e na descrição e foco nas pessoas e no comportamento informacional. No entanto, consideramos que o planejamento da instituição e seus aspectos administrativos e tecnológicos estão diretamente relacionados à eficiência dos recursos informacionais.

Perspectivas em Gestão \& Conhecimento, João Pessoa, v. 10, número especial, p. 23-37, mar. 2020. 


\section{PROCEDIMENTOS METODOLÓGICOS}

Para que um trabalho seja caracterizado como científico, deve ser aceito pelos pares e seguir uma metodologia que norteie os procedimentos aplicados. Salomon (1977) indica a necessidade de alguns requisitos para que um trabalho tenha esse teor e rigor científico: a atividade deve produzir ciência, ser produto dela ou tê-la como modelo. Portanto, o percurso metodológico é um conjunto de ações desenvolvidas para atribuir sentido à pesquisa e tornála harmoniosa e coesa. Silva (2005) esclarece que é preciso adotar uma metodologia científica como um elemento norteador do trabalho, ou seja, o espaço em que são explicados os meios para se chegar ao resultado obtido, os instrumentos utilizados para tal feito e a definição do campo de estudo (CASIMIRO, 2019).

No que concerne aos aspectos metodológicos desta pesquisa, podemos caracterizá-la como um estudo exploratório, descritivo e indutivo. Sampiere, Collado e Lúcio (2006, p. 99) definem o primeiro como um estudo que possibilita "examinar um tema ou problema de pesquisa pouco estudado, a respeito do qual se tem muitas dúvidas ou não foi abordado antes"; o segundo (p. 100) "consiste em descrever situações, acontecimentos e feitos, isto é, dizer como se manifesta determinado fenômeno"; e o terceiro, como um (p.82) "processo mental por intermédio do qual, partindo de dados particulares, suficientemente constatados, infere-se uma verdade geral ou universal".

Trata-se, pois, de uma pesquisa fundamentalmente de abordagem qualitativa e de natureza documental direta, que Lakatos e Marconi (2017) conceituam como um estudo em que, durante a fase de levantamento de dados, são escolhidos documentos de fonte primária. A abordagem qualitativa agrega valor aos resultados da pesquisa por ter uma ótica mais flexível, de modo a se adaptar à fenomenologia do objeto de estudo. Assim, de modo mais indutivo, analisa os dados e a atribuição de significados. Para este estudo, fizemos as buscas documentais na fundamentação teórica sobre Smart Campus e Gestão da Informação, bem como nas informações presentes nas Portarias no 84 e 85, de 10 de junho de 2019, da Universidade Federal de Campina Grande, que regulamentam o Smart Campus nessa instituição.

Para analisar os dados, adotamos a técnica de Análise de Conteúdo proposta por Laurence Bardin (2010). Para esse tipo de análise, as informações e os dados contidos no texto são meios que o sujeito emprega para se expressar, e o pesquisador que o analisar terá a função de criar núcleos de sentido baseados nos termos que se repetem e inferir uma expressão que as represente, criando categorias que, para este estudo, são as etapas de GI propostas por Choo (2003). A autora indica que a análise de conteúdo acontece, geralmente, em um processo contínuo de três fases: a da pré-análise, a da exploração do material e a do tratamento dos resultados e sua interpretação.

Nesta pesquisa, na fase de pré-análise, traçamos os objetivos e criamos a metodologia, tomando como base as vivências e as leituras realizadas anteriormente. Na fase de exploração do material, procedemos à leitura criteriosa do material para criar categorias compatíveis com a pesquisa, a fim de identificar os padrões do conteúdo estudado. Quanto ao tratamento dos resultados e sua interpretação, fizemos a tabulação bruta e escolhemos as vertentes que mais representavam a pesquisa. Também tecemos as análises do assunto abordado.

Com os resultados, criamos núcleos de sentido adaptados às etapas de $\mathrm{Gl}$ de Choo (2003) e o Quadro 3, que facilitam a interpretação e a contextualização de cada fator. Essas interpretações são influenciadas pela leitura realizada imperativamente na primeira fase e nas fases seguintes (BARDIN, 2010).

No Fórum Nacional de Pró-Reitores de Planejamento e de Administração das Instituições Federais de Ensino Superior (FORPLAD), ocorrido no período de 17 a 19 de junho

Perspectivas em Gestão \& Conhecimento, João Pessoa, v. 10, número especial, p. 23-37, mar. 2020. 
de 2019, na cidade de Campina Grande (Paraíba), o prefeito da UFCG, através de uma enquete, identificou as universidades federais que implantaram um Smart Campus no Brasil. No Nordeste, só a Universidade Federal do Rio Grande do Norte (em 2016) e a UFCG (em 2019) criaram um Smart Campus. Em outras regiões, temos a Universidade Federal do Espírito Santo (em 2016) e a Universidade Federal de Santa Maria (em 2016). Portanto, devido à facilidade de acesso ao gestor do modelo da UFCG - o prefeito universitário - a instituição foi escolhida como objeto de análise da pesquisa.

Para complementar a coleta de dados bibliográfica, aplicamos um questionário ao gestor do projeto de implantação do Smart Campus na UFCG, que é o prefeito da instituição, pois o Smart Campus da UFCG foi desenvolvido e implantado de acordo com a Portaria no 85 , de 10 de junho de 2019, como um setor que compõe a estrutura organizacional da Prefeitura Universitária.

Quadro 3 - Questionário e relação com a GI

\begin{tabular}{|c|c|}
\hline $\begin{array}{l}\text { Etapas do modelo de } \\
\text { Choo (2003) }\end{array}$ & Perguntas do questionário \\
\hline \multirow{2}{*}{$\begin{array}{l}\text { Identificação das } \\
\text { necessidades de } \\
\text { informação }\end{array}$} & $\begin{array}{l}\text { 1. Como a UFCG busca compreender as necessidades de informação da } \\
\text { comunidade? (Ex.: sistema, estudo de usuário, estatística) }\end{array}$ \\
\hline & 2. Os usuários ou colegas registram as ideias dadas? Se o fazem, como? \\
\hline \multirow[t]{2}{*}{ Aquisição de informação } & $\begin{array}{l}\text { 3. Para desempenhar as atividades de gestão, os profissionais utilizam } \\
\text { alguma fonte para obter a informação? Se sim, quais? (Ex.: gestor, } \\
\text { colegas, e-mail, redes sociais, buscadores, legislação manual). }\end{array}$ \\
\hline & $\begin{array}{l}\text { 4. Quais os processos técnicos realizados pela UFCG para viabilizar o } \\
\text { Smart Campus? }\end{array}$ \\
\hline $\begin{array}{l}\text { Organização e } \\
\text { armazenamento da } \\
\text { informação }\end{array}$ & $\begin{array}{l}\text { 5. Há uma preocupação em atualizar os recursos tecnológicos e investir } \\
\text { neles? Quais? (Ex.: atualização do sistema, capacitação do pessoal) }\end{array}$ \\
\hline \multirow{2}{*}{$\begin{array}{l}\text { Desenvolvimento de } \\
\text { produtos informacionais e } \\
\text { serviços }\end{array}$} & $\begin{array}{l}\text { 6. Quais os serviços que a UFCG presta à comunidade no que concerne } \\
\text { ao Smart Campus? }\end{array}$ \\
\hline & $\begin{array}{l}\text { 7. Quais são os produtos que a UFCG oferece à comunidade, no que } \\
\text { concerne ao Smart Campus? (Ex.:manual de conduta ou de serviços } \\
\text { prestados pela universidade, folders, folhetos descritivos) }\end{array}$ \\
\hline \multirow[t]{2}{*}{ Distribuição da informação } & $\begin{array}{l}\text { 8. Quais os canais de disseminação da informação são adotados para } \\
\text { manter a relação entre os funcionários diretamente ligados à aplicação } \\
\text { do Smart Campus e os gestores da UFCG? (Ex.: reuniões, exposições de } \\
\text { motivos, relatório de atividades) }\end{array}$ \\
\hline & $\begin{array}{l}\text { 9. A UFCG dispõe de algum local para divulgar notícias? (Ex.: fan page, } \\
\text { blog, redes sociais) }\end{array}$ \\
\hline Uso da informação & $\begin{array}{l}\text { 10. A UFCG desempenha alguma ação para identificar a eficácia do uso da } \\
\text { informação que disponibiliza? (Ex.: pesquisa de satisfação, conversas } \\
\text { informais) }\end{array}$ \\
\hline
\end{tabular}

Fonte: Elaboração própria (2019)

O instrumento foi constituído de dez perguntas abertas (elencadas no Quadro 3), com foco nas etapas de $\mathrm{GI}(\mathrm{CHOO}, 2003)$ e as aplicabilidades de Smart Campus nessa instituição, de acordo com o exposto nas Portarias № 84 e 85 de 10 de junho de 2019 (UNIVERSIDADE FEDERAL DE CAMPINA GRANDE, 2019). As respostas do gestor indicaram algumas técnicas de aplicação do Smart Campus na UFCG até o momento.

Perspectivas em Gestão \& Conhecimento, João Pessoa, v. 10, número especial, p. 23-37, mar. 2020. 


\section{SMART CAMPUS E GI NA UFCG: ANÁLISES}

Seguindo o referencial teórico desta pesquisa, escolhemos o modelo de Gl que mais bem se adaptasse às necessidades e às aplicabilidades necessárias ao Smart Campus. Assim, escolhemos o modelo de Choo (2003), porque tem seis etapas que podem englobar essa exigência, é amplamente utilizado e indicado pela literatura em Ciência da Informação, em Gestão e em Administração Pública e é um modelo com alto grau adaptabilidade aos princípios descritos nas Portarias no 84 e 46 de 10 de junho de 2019 da UFCG (UNIVERSIDADE FEDERAL DE CAMPINA GRANDE, 2019).

Com base nas seis etapas do modelo de Choo (2003), nas Portarias no 84 e 46, de 10 de junho de 2019 da UFCG, e nas respostas recebidas do questionário ao gestor responsável pela implantação do Smart Campus na UFCG, foi possível identificar alguns pontos de sentido e relação importantes, a saber:

- Etapa 1 - Identificação das necessidades de informação: Existe um sistema de captação de informação por meio do qual é possível identificar as necessidades dos usuários com a sistematização das demandas formais e informais que geram um banco de dados. "Além disso, o Smart Campus tem mantido relação estreita com professores que têm a intenção de utilizar o campus como um campo experimental (laboratório). Assim, a relação com os alunos acontece de forma direta".

- Etapa 2 - Aquisição da informação: Para adquirir informações do cotidiano, o gestor e os demais membros da instituição adotam como ferramenta as redes sociais do tipo mensagem eletrônica. "As redes sociais, através de grupos de profissionais que desempenham funções semelhantes, buscadores, sistemas governamentais (Ex. Painel de Preços), consulta jurídica à Procuradoria Federal etc."

O gestor usou a ferramenta benchmarking para identificar e comparar um modelo de Smart Campus no Brasil antes de iniciar a implantação do modelo na UFCG. Em sua fala, ele referiu:

Foi realizado uma visita técnica ao Campus da FACENS em Sorocaba, onde conhecemos o funcionamento do Smart Campus, em seguida houve uma análise da realidade da UFCG onde foram identificados projetos em andamento que poderiam ser agrupados para iniciar o Smart Campus da UFCG e a criação de uma comissão para estabelecer o modelo a ser implantado na UFCG.

Além disso, foram desenvolvidas pesquisas relacionadas ao tema a inclusive em nível nacional no Fórum de Pro-Reitores de Planejamento e Administração das Universidades Federais.

- Etapa 3 - Organização e armazenamento da informação: O gestor demonstrou grande interesse pela captação e pela segurança dos dados no Smart Campus, considerando que eles são os elementos estratégicos nesse tipo de plataforma. Sobre isso, falou:

Existe a preocupação com a infraestrutura de dados da UFCG tendo em vista a expectativa de que sejam acrescentados diversos sensores à rede. Assim, a perspectiva é de que seja criada uma rede exclusiva para os sensores. Além disso, existe preocupação com a quantidade de informação que será armazenada por projeto, já que se quer criar um histórico de dados para os diversos temas que serão objeto de estudo no Smart Campus. Entretanto, não foi necessário realizar investimento neste aspecto até este momento.

- Etapa 4 - Desenvolvimento de produtos e serviços de informação: A UFCG apresentou um conjunto de informações importantes que estão disponíveis para os usuários acessarem e

Perspectivas em Gestão \& Conhecimento, João Pessoa, v. 10, número especial, p. 23-37, mar. 2020. 
outras em desenvolvimento. Esse serviço favorece a transparência da gestão. A esse respeito, relatou:

\begin{abstract}
Foram desenvolvidos sistemas de gestão para os serviços de água e energia, além disso está sendo montado o sistema que apresentará as informações coletadas pelos sensores (inclusive os de água e energia) para a comunidade, tanto na página do Smart Campus como na sede do Smart Campus. Outras informações, em nível mais detalhado, estarão acessíveis apenas ao pessoal de interesse (ex.: manutenção, gestores). Além disso, o Smart Campus se constitui em uma plataforma que aproxima a comunidade da gestão à medida em que ela própria está engajada na busca de soluções para os problemas do seu dia a dia.
\end{abstract}

- Etapa 5 - Distribuição da informação: $O$ gestor adotou uma forma de distribuir a informação mais próxima das pessoas em encontros semanais com diversos grupos dentro da instituição. "Atualmente a informação está sendo disseminada apenas através de reuniões. A UFCG divulga suas notícias em sua página na internet e mantém um perfil oficial no Instagram".

- Etapa 6 - Uso da informação: $O$ acompanhamento e as avaliações sobre o desempenho ainda estão sendo estruturados, porém já existem iniciativas em andamento para identificar as necessidades do público-alvo. Segundo o gestor,

não existe uma ação formal de avaliação de feedback, entretanto a própria equipe de assessoria de comunicação, através de um processo de autoavaliação sentiu a necessidade de reformular, recentemente, a Homepage e passar a divulgar conteúdos mais atrativos pelo perfil oficial do Instagram. Este último, pela natureza da plataforma, permitiu avaliar que o engajamento cresceu com a mudança no conteúdo postado.

Esse cenário evidencia que as aplicabilidades do Smart Campus dialogam com o modelo de $\mathrm{Gl}$, que se preocupa em identificar as necessidades, o que resulta na construção de um banco de dados e na relação direta entre os atores envolvidos. A informação é adquirida por meio da interação entre professores e alunos em redes sociais. Logo, a transparência da gestão é orientada pelo desenvolvimento de produtos e serviços de informação. A informação é distribuída nas reuniões semanais, com grupos de interesses, e divulgada na página da internet e nas redes sociais. Quanto ao uso da informação, faltam e monitoramento e avaliações sobre o desempenho. Nesse contexto, existem iniciativas em andamento.

Observamos que a GI facilita o controle dos processos, porém é essencial monitorar e apresentar indicadores que subsidiem a tomada de decisão para melhorar as práticas informacionais. Portanto, é essencial enfatizar a tecnologia da informação com foco na mensuração desses resultados qualitativos e quantitativos. Para Dias e Beluzzo (2003), a GI "é um conjunto de conceitos, princípios, métodos e técnicas utlizadas na prática administrativa e colocada em execução pela liderar um Serviço de Informação". Então, consideramos as aplicabilidades do Smart Campus como um serviço de informação que, para os autores, tem como característica principal a prestação de serviços à comunidade e à sociedade em geral.

No contexto das instituições, embora a tecnologia da informação só seja usada internamente, é um suporte essencial para se prestar um serviço de informação de boa qualidade, a partir da eficiência na recuperação, no monitoramento e na apresentação de indicadores de informação em um ciclo contínuo de produção, acesso e uso com dados previamente estruturados.

Atualmente a UFCG está com os seguintes projetos em funcionamento:

- SIGA: gestão do uso de água no campus;

Perspectivas em Gestão \& Conhecimento, João Pessoa, v. 10, número especial, p. 23-37, mar. 2020. 
- SCIKE: gestão do uso de energia elétrica;

- Monitoramento de acesso: contagem do acesso de veículos através do reconhecimento de imagens;

- Dashboard: interface comum dos projetos do Smart Campus.

O Smart Campus é um modelo promissor para se aplicar a internet das coisas na gestão universitária e na socialização dos recursos em tempos de crise econômica. Trata-se de um modelo cuja aplicabilidade está em andamento, porém, com grande probabilidade de ser replicado nas 63 IFES existentes no Brasil. É prematuro concluir a eficácia ou o impacto do modelo por ser ainda emergente, assim como o próprio conceito de Smart Campus.

Ressalte-se, entretanto, que a aplicabilidade das tecnologias envolvidas nesse modelo tem um alto potencial para reduzir custos e aumentar a sustentabilidade e a aprendizagem. Exemplo disso é o monitoramento, em tempo real, do uso das salas de aula e dos seus recursos. Em outras palavras, se o professor ficar uma hora na sala que estava reservada para ele por duas horas, o sistema de sensor detectará sua ausência e a dos estudantes e cortará os recursos de energia elétrica daquele ambiente, para impedir que outras pessoas usem aquele ambiente no intervalo reservado para determinado docente. Isso também ficaria registrado no sistema de gestão dos espaços, gerando o perfil histórico de cada usuário. Em outro cenário, um estudante pode seguir as aulas teóricas em uma plataforma de ensino a distância, e as aulas práticas, nos laboratórios ou nas organizações. Esses recursos ainda não são aplicados efetivamente na UFCG. São exemplos de controle em um ambiente inteligente, que a gestão se preocupa em implantar na organização objeto desta pesquisa.

Outro exemplo é o fato de o estudante morar a $200 \mathrm{~km}$ da universidade e poder estudar, nos dois primeiros anos do curso, a distância. Para isso, precisa ir para o campus quando estiver apto a atuar em um laboratório com supervisão ou em um estágio para cumprir as horas práticas da formação. Então, como não esperar que o uso das tecnologias mencionadas poderá elevar o nível gerencial e educacional das instituições de ensino? O Smart Campus é uma realidade no mundo, porém, no Brasil, ainda apresenta poucas iniciativas nesse sentido, mas isso não impede que se vislumbre um futuro promissor, caso o modelo consiga contemplar a tecnologia, a gestão, o ensino e as pessoas.

\section{CONSIDERAÇÕES FINAIS}

As organizações modernas, como Amazon, Google, Tesla etc., usam tecnologias inteligentes no processo interno de aprimoramento das rotinas administrativas. Isso ajuda os profissionais a colaborarem entre si e a se dedicarem no desenvolvimento de ideias. Esses ambientes são basicamente espaços onde se usa um conjunto de tecnologias integradas para facilitar ou aumentar o desempenho das atividades ou da vida das pessoas. A integração, sobretudo da informação, seja sobre tecnologias, clientes, concorrentes e outros, passa a ser mais compartilhada com o uso das TICs.

Em um cenário de Big Data, como é o caso do Smart Campus, a Ciência da Informação tem muito a contribuir, e a gestão da informação é uma delas. $O$ alto volume de dados gerados nessas plataformas demanda, além dos algoritmos para gerenciar a informação, uma política de Gestão da Informação. O posicionamento do gestor é fundamental na fusão entre a tecnologia e as necessidades do usuário. Entende-se que o processo de tomada de decisão requer informações específicas para auxiliar o gestor, o que remete a um processo integrado de criação, organização, disseminação e uso da informação de forma transparente e controlada no âmbito da organização.

Acreditamos que, como a emissão das Portarias no 84 e 85, de 10 de junho de 2019, que viabilizam o Smart Campus na UFCG é recente, as aplicabilidades das diretrizes ainda estão

Perspectivas em Gestão \& Conhecimento, João Pessoa, v. 10, número especial, p. 23-37, mar. 2020. 
em fase embrionária, portanto, é necessário proporcionar um tempo maior para que a instituição se estabeleça como campo e que, caso ocorra o investimento correto em parceria com gestores, técnicos administrativos, professores e os demais membros da comunidade universitária, a UFCG tem grande potencial para se tornar uma referência na aplicação dessa técnica no país.

O objetivo do artigo foi alcançado, porquanto foi possível identificar a maneira como a UFCG gerencia sua informação. O desenvolvimento de um plano mais detalhado e específico de GI poderia ser sugerido, porém, por se tratar de um modelo que apresenta várias dimensões em desenvolvimento, não se pode concluir que ele não irá incorporar os elementos essenciais para a GI.

Os conceitos, os modelos e as teorias reconhecidos pelos atores envolvidos nesse processo contribuem para o desenvolvimento de ações de planejamento, controle e avaliação das práticas informacionais. Além disso, é preciso compreender as condições em que a informação é criada na instituição e as estruturas específicas de gestão. $O$ estudo confirma as questões culturais de tecnologia da informação e de disseminação e de acesso e uso que são fatores críticos para a eficiência dos processos informacionais. Compete às instituições gerirem e monitorarem esses processos e, sobretudo, compreender o comportamento do usuário e suas necessidades informacionais.

Apesar da implantação do uso de ferramentas inteligentes na gestão do campus, percebem-se, na prática, alguns gargalos ligados à gestão da informação. Essa constatação fornece elementos a serem considerados pelos gestores no desenvolvimento dos modelos de gestão que estão sendo aprimorados para o campus inteligente. Em outras palavras, na prática, é preciso haver uma combinação entre a gestão da informação, a administração e a Ciência da Computação na gestão do campus inteligente. As pesquisas sobre o assunto ainda são escassas, e os trabalhos se concentram nas ciências puras. Este trabalho surgiu da esperança de aumentar a discussão e de se desenvolverem mais pesquisas sobre o assunto na perspectiva da Ciência da Informação e da Administração.

No que concerne à limitação deste estudo, apontamos o fato de o tema ser tão novo que não há pesquisas sobre a aplicabilidade do Smart Campus em outras instituições brasileiras, o que dificulta comparar as melhorias das universidades que usam esse método e as que não o utilizam. Mas compreendemos que essa limitação será atenuada com o tempo e com mais trabalhos sobre o assunto.

Finalizamos este artigo com a certeza de ter alcançado o objetivo proposto, entretanto, sem a intenção de esgotar o tema, mas de lançar um olhar para as novas questões que emergem sobre o assunto. Afinal, quando uma pergunta é respondida, a curiosidade inerente ao ator de pesquisar é aflorada para que se questione sobre outros pontos de vista.

\section{REFERÊNCIAS}

Ai-Min et al. Situational Awareness System in the Smart Campus. Anais [...]. IEEE Access, 2018.

ARAÚJO, Carlos Alberto Ávila. Fundamentos da Ciência da Informação: correntes teóricas e o conceito de informação. Perspectivas em Gestão \& Conhecimento, João Pessoa, v. 4, n. 1, p.5779, jan./jun. 2014.2 Disponível em http://www.periodicos.ufpb.br/ojs/index.php/pgc/article/view/19120/10827. Acesso em 20 set. 2019.

Perspectivas em Gestão \& Conhecimento, João Pessoa, v. 10, número especial, p. 23-37, mar. 2020. 
CASIMIRO, Adelaide Helena Targino. Gestão por Competências nos Cursos de Arquivologia no Brasil: abordagem nos princípios da Gestão do Conhecimento. 2019. Dissertação (Mestrado em Ciência da Informação) - Universidade Federal da Paraíba, João Pessoa, 2019.

CHOO, Chun Wei. A organização do conhecimento: como as organizações usam a informação para criar significado, construir conhecimento e tomar decisões. São Paulo: Senac, 2003.

DAVENPORT, Thomas Hayes. Ecologia da informação. São Paulo: Futura, 2002.

DIAS, M.M.K.; BELUZZO, R.C.B. Gestão da informação em ciência e tecnologia sob a ótica do cliente. Bauru: EDUSC, 2003.

DONG, Xin; KONG, Xiangjie; ZHANG, Fulin et al. On Campus: a mobile platform towards a smart campus. SpringerPlus. 2016.

DRUCKER, Peter F. The coming of the new organization. Harvard Business Review, n. 66, jan./fev. 1988. Disponível em https://hbr.org/1988/01/the-coming-of-the-new-organization. Acesso em 14 out. 2019.

FENG, Nan; YINA, Suo; XUEYONG, Jia et al. Real-Time Monitoring of Smart Campus and Construction of Weibo Public Opinion Platform. Anais [...]. IEEE Access, 2018.

KAR, A.; GUPTA, M. P. How to make a smart campus: Smart Campus Programme in IIT Delhi. 2015.

KWOK, Lam-for. A vision for the development of i-campus lam for work. Smart Learning environments. 2015.

LE COADIC, Yves-François. A Ciência da Informação. Brasília: Briquet de Lemos. 2004.

LEITE, Fernando César Lima. Modelo genérico de gestão da informação científica para instituições de pesquisa na perspectiva da comunicação científica e do acesso aberto. 2011. 262 f. Tese (Doutorado em Ciência da Informação) - Universidade de Brasília, Brasília, 2011. Disponível em: http://repositorio.unb.br/handle/10482/9753. Acesso em 01 out. 2019.

LI, Luo. Data acquisition and analysis of Smart Campus based on wireless sensor. Wireless Personal Communications. 2018.

Liang, Yi; Chen, Zixi, Intelligent and Real-Time Data Acquisition for Medical Monitoring in Smart Campus. Anais [...]. IEEE Access. 2018.

MCGEE, John Vernon; PRUSAK, Laurence. Gerenciamento estratégico da informação: aumente a competitividade e a eficiência de sua empresa utilizando a informação como uma ferramenta estratégica. Rio de Janeiro: Campus, 1994.

Qu, Shaojie; Li, Kan; Zhang, Shuhui; Wang, Yongchao. Predicting Achievement of Students in Smart Campus. Anais... IEEE Access. 2018.

Perspectivas em Gestão \& Conhecimento, João Pessoa, v. 10, número especial, p. 23-37, mar. 2020. 
ROMANO, R. R.; SIRON, L. G. P. P.; PACHECO, C. P. Smart Campus FACENS: construindo uma cidade inteligente em um Campus Universitário utilizando-se do FabLab. 2016. Disponível em http://fablearn.org/wp-content/uploads/2016/09/FLBrazil2016paper150.pdf. Acesso em 26 set. 2019.

STEWART, T. A. Capital intelectual: a nova vantagem competitiva das empresas. Rio de Janeiro: Campus, 1998.

UNIVERSIDADE FEDERAL DE CAMPINA GRANDE. Boletim de Serviço: portarias no 84 e 85 de 10 de junho de 2019. Campina Grande: Reitoria, 2019. Disponível em https://portal.ufcg.edu.br/phocadownload/userupload/Boletim de servico/boletim\%20de\%2 0servio\%20-\%202019-30.pdf. Acesso em: 07 nov. 2019.

XIN, Xu; YUNSHENG, Wang; SHUJIANG, Yu. Teaching performance evaluation in Smart Campus. Anais [...]. IEEE Access. 2018.

YANG, Ai-Min; LI, Shan-Shan; REN, Cui Huan et al. Situational Awareness System in the Smart Campus. Anais [...]. IEEE Access, 2018.

YING, Chen; ZHANG, Runtong; SHOUYI, Zhang et al. Service encapsulation-based model for Smart Campus. Journal of Electronic Commerce in Organizations, 2012.

ZHIHONG, Tian; YU, Cui; LUN, An et al. A Real-Time Correlation of Host-Level Events in Cyber Range Service for Smart Campus. Anais [...]. IEEE Access, 2018.

Artigo recebido em 20/11/2019 e aceito para publicação em 25/02/2020

Perspectivas em Gestão \& Conhecimento, João Pessoa, v. 10, número especial, p. 23-37, mar. 2020. 